\title{
Glutathione-dependent formaldehyde dehydrogenase/GSNO reductase from Arabidopsis. Expression pattern and functional implications in phytoremediation and pathogenesis
}

\author{
M Carmen Martínez*, Maykelis Díaz, Hakima Achkor and M Carme Espunya
}

Address: Departamento de Bioquímica y Biología Molecular, Facultad de Ciencias, Universidad Autónoma de Barcelona, 08193 Bellaterra

(Barcelona), Spain

Email: M Carmen Martínez* - carmen.martinez@uab.es

* Corresponding author

from Cell Biology of Nitric Oxide and Cell Death in Plants

Yalta, Ukraine, 8-II September 2004

Published: 3I May 2005

BMC Plant Biology 2005, 5(Suppl I):S22 doi:I0.I I86/I47|-2229-5-SI-S22

The glutathione-dependent formaldehyde dehydrogenase (FALDH) is the main enzyme of the formaldehyde detoxification system in eukaryotes. In Arabidopsis FALDH is coded by a single gene, which is constitutively expressed [1]. By immunolocalization experiments on Arabidopsis root and leaf sections, we demonstrate that the pattern of expression of the enzyme is cell specific. By using tobacco BY-2 cell cultures we show that FALDH co-localizes with tubulin on the cortical microtubules and the microtubules figures (preprophase band, mitotic spindle and phragmoplast), which suggests a role for FALDH in some plant-specific function during cell division. Overexpression of FALDH in Arabidopsis plants results in a $25 \%$ increase in the efficiency of elimination of exogenous formaldehyde, whereas plants with reduced levels of FALDH, bearing antisense constructs, show a reduced ability and slower rate in formaldehyde elimination [2]. These results confirm the central role of FALDH in formaldehyde metabolism in plants and have important implications in the phytoremediation of environmental formaldehyde.

The importance of FALDH has been greatly increased by the discovery of its potent activity toward S-nitrosoglutathione, the condensation product of glutathione and nitric oxide (NO) [3-5]. NO and NO-related metabolites, such as S-nitrosothiols (SNOs) play a central role in signal transduction and host defense [6]. We have investigated the gene response to mechanical wounding and plant hormones involved in the signal transduction pathway, showing that the gene is down-regulated by wounding in a JA-dependent pathway, and that it is transcriptionally activated by salicylic acid [7]. This is the first time that reg- ulation of FALDH in response to signals associated with plant defense has been demonstrated.

\section{References}

I. Martínez MC, Achkor H, Persson B, Fernández MR, Shafqat J, Farrés J, Jörnvall H, Parés X: Arabidopsis formaldehyde dehydrogenase: molecular properties of plant class III alcohol dehydrogenase provide further insights into the origins, structure and function of plant class $\mathbf{P}$ and liver class I alcohol dehydrogenases. Eur J Biochem 1996, 241:849-857.

2. Achkor H, Díaz M, Fernández MR, Biosca JA, Parés X, Martínez MC: Enhanced formaldehyde detoxification by overexpression of glutathione-dependent formaldehyde dehydrogenase from Arabidopsis thaliana. Plant Physiol 2003, 132:2248-2255.

3. Jensen DE, Belka GK, Du Bois GC: S-Nitrosoglutathione is a substrate for rat alcohol dehydrogenase class III isoenzyme. Biochem J 1998, 331:659-668.

4. Liu L, Hausladen A, Zeng M, Que L, Heitman J, Stamler JS: A metabolic enzyme for S-nitrosothiol conserved from bacteria to humans. Nature 200I, 41 0:490-494.

5. Sakamoto A, Ueda M, Morikawa H: Arabidopsis glutathionedependent formaldehyde dehydrogenase is an S-nitrosoglutathione reductase. FEBS Lett 2002, 5 | 5:20-24.

6. Wendehenne D, Pugin A, Klessig DF, Durner J: Nitric oxide: comparative synthesis and signaling in animal and plant cells. Trends Plant Sci 200I, 6: 177-I83.

7. Delledonne M, Xia Y, Dixon RA, Lamb C: Nitric oxide functions as a signal in plant disease resistance. Nature 1998, 394:585-588.

8. Huang X, Stettmaier K, Michel C, Hutzler P, Mueller MJ, Durner J: Nitric oxide is induced by wounding and influences jasmonic acid signaling in Arabidopsis thaliana. Planta 2004, 2 I 8:938-946.

9. Díaz M, Achkor H, Titarenko E, Martínez MC: The gene encoding glutathione-dependent formaldehyde dehydrogenasel GSNO reductase is responsive to wounding, jasmonic acid and salicylic acid. FEBS Letters 2003, 543:136-139. 